\title{
Dynamic and weighted stabilizations of the $L$-scheme applied to a phase-field model for fracture propagation
}

\author{
Christian Engwer, Iuliu Sorin Pop, Thomas Wick
}

\begin{abstract}
We consider a phase-field fracture propagation model, which consists of two (nonlinear) coupled partial differential equations. The first equation describes the displacement evolution, and the second is a smoothed indicator variable, describing the crack position. We propose an iterative scheme, the so-called $L$-scheme, with a dynamic update of the stabilization parameters during the iterations. Our algorithmic improvements are substantiated with two numerical tests. The dynamic adjustments of the stabilization parameters lead to a significant reduction of iteration numbers in comparison to constant stabilization values.
\end{abstract}

\section{Introduction}

This work is an extension of [3] in which an L-type iterative scheme (see [5, 8]) with stabilizing parameters for solving phase-field fracture problems was proposed. In [3], the stabilization parameters were chosen as constants throughout an entire computation. With these choices, the convergence of the scheme has been proven rigorously. The resulting approach performs well in the sense that an unlimited number of iterations compared to a truncated scheme yields the same numerical solution. The results were validated by investigating the load-displacements curves. Moreover, the robustness of the scheme w.r.t. spatial mesh refinement was shown.

Christian Engwer

Institut für Numerische und Angewandte Mathematik, Universität Münster, Einsteinstrasse 62, 48149 Münster, Germany, e-mail: christian. engwer@uni-muenster.de

Iuliu Sorin Pop

Universiteit Hasselt, Faculty of Sciences, Agoralaan Gebouw D - B-3590 Diepenbeek, Belgium, e-mail: sorin.pop@uhasselt.be

Thomas Wick

Leibniz Universität Hannover, Institut für Angewandte Mathematik, Welfengarten 1, 30167 Hannover, Germany, e-mail: thomas.wick@ifam.uni-hannover.de 
Nonetheless, the iteration numbers (for an unlimited number of iterations) remained high.

In this work, we propose and compare two extensions of the aforementioned scheme. First, we update the $L$ scheme parameters dynamically. Second, we use an adaptive weight depending on the fracture location inside the domain. For the latter idea, we use the phase-field variable to weight $L$ locally.

The outline of this work is as follows: In Section 2 the model is stated whereas Section 3 presents the dynamic choice of the stablization parameters. In Section 4 . we present two numerical tests to study the performance of the proposed scheme.

\section{The phase-field fracture model}

We consider the crack propagation model proposed in [3]. Let $\Omega \subset \mathbb{R}^{d}$ be a $d$ dimensional, polygonal and bounded domain and $T>0$ a maximal time. We use common notations, and in particular $W^{1, \infty}(\Omega)$ denotes the space of functions on $\Omega$ having essentially bounded weak derivatives in any direction, while $H_{0}^{1}(\Omega)$ contains the functions having square integrable weak derivatives, and vanishing at the boundary of $\Omega$ (in the sense of traces). For the ease of writing, we let $V:=H_{0}^{1}(\Omega)^{d}$ and $W:=W^{1, \infty}(\Omega)$. For (almost every) location $x \in \Omega$ and time $t \in(0, T]$ the vectorvalued displacements are denoted by $u$. The fracture and its propagation within $\Omega$ are modeled with the help of a phase field variable $\varphi$, which approximates the characteristic function of the intact region of $\Omega$. Written in weak form, the fracture propagation model in resumes to finding $(u(t), \varphi(t)) \in V \times W:=\left(H_{0}^{1}(\Omega)\right)^{d} \times W^{1, \infty}(\Omega)$ such that for $t \in(0, T]$ :

- Step 1: given $\left(u^{n, i-1}, \varphi^{n, i-1}\right)$ find $u^{n, i}$ such that $a_{u}\left(u^{n, i}, v\right):=L_{u}\left(u^{n, i}-u^{n, i-1}, v\right)+\left(g\left(\varphi^{n, i-1}\right) \sigma^{+}\left(u^{n, i}\right), \mathbf{e}(v)\right)+\left(\sigma^{-}\left(u^{n, i}\right), \mathbf{e}(v)\right)=0$,

$\forall v \in V_{h}$,

- Step 2: given $\left(\varphi^{n, i-1}, u^{n, i}, \varphi^{n-1}\right)$ find $\varphi^{n, i}$ such that

$$
\begin{aligned}
& a_{\varphi}\left(\varphi^{n, i}, \psi\right):=L_{\varphi}\left(\varphi^{n, i}-\varphi^{n, i-1}, \psi\right)+G_{c} \varepsilon\left(\nabla \varphi^{n, i}, \nabla \psi\right)-\frac{G_{c}}{\varepsilon}\left(1-\varphi^{n, i}, \psi\right) \\
& \quad+(1-\kappa)\left(\varphi^{n, i} \sigma^{+}\left(u^{n, i}\right): \mathbf{e}\left(u^{n, i}\right), \psi\right)+\left(\Xi+\gamma\left[\varphi^{n, i}-\varphi^{n-1}\right]^{+}, \psi\right)=0, \\
& \forall \psi \in W_{h} .
\end{aligned}
$$

Here, we note that the 'time' $t$ appears only the irreversibility constraint $\partial_{t} \varphi \leq 0$, yielding an incremental problem and which is regularized using an augmented Lagrangian penalization $\left(\Xi+\gamma\left[\varphi^{n, i}-\varphi^{n-1}\right]^{+}, \psi\right)$ as proposed in $[9]$. Here, $\Xi$ is an $L^{2}(\Omega)$ function and $\gamma$ a positive parameter.

Furthermore, in the above, $\varepsilon$ is a (small) phase-field regularization parameter, $G_{c}>0$ is the critical elastic energy restitution rate, and $0<\kappa \ll 1$ is a regularization parameter used to avoid the degeneracy of the elastic energy. The latter is similar 
to replacing the fracture with a softer material. Next, $g(\varphi):=(1-\kappa) \varphi^{2}+\kappa$ is the degradation function, and $\mathbf{e}:=\frac{1}{2}\left(\nabla u+\nabla u^{T}\right)$ is the strain tensor.

The stress tensor in the above is split into a tensile and compressive part,

$$
\sigma^{+}:=2 \mu_{s} \mathbf{e}^{+}+\lambda_{s}[\operatorname{tr}(\mathbf{e})]^{+} I, \quad \sigma^{-}:=2 \mu_{s}\left(\mathbf{e}-\mathbf{e}^{+}\right)+\lambda_{s}\left(\operatorname{tr}(\mathbf{e})-[\operatorname{tr}(\mathbf{e})]^{+}\right) I,
$$

where $[\cdot]^{+}$stands for the positive cut of the argument. Further, $\mathbf{e}^{+}=\mathbf{P} \mathbf{\Lambda}^{+} \mathbf{P}^{T}$, with $\mathbf{P}$ being the matrix containing the unit eigenvectors corresponding to the eigenvalues of the strain tensor $\mathbf{e}$. In particular, for $d=2$ one has $\mathbf{P}=\left[v_{1}, v_{2}\right]$ and

$$
\boldsymbol{\Lambda}^{+}:=\boldsymbol{\Lambda}^{+}(u):=\left(\begin{array}{cc}
{\left[\lambda_{1}(u)\right]^{+}} & 0 \\
0 & {\left[\lambda_{2}(u)\right]^{+}}
\end{array}\right) .
$$

\section{The $L$-scheme with dynamic updates of the stabilization parameters}

The iteration (17)-(2) is essentially the scheme proposed in [3], in which the stabilization parameters $L_{u}$ and $L_{\varphi}$ are taken constant. To improve the convergence behaviour of the scheme, we propose a dynamic update of these parameters.

Dynamic update at each iteration / constant in space: The iteration discussed in [3] uses constant parameters $L_{u}$ and $L_{\varphi}$. With this choice, the convergence has been proved rigorously. However, the number of iterations can remain high. High iteration numbers for phase-field fracture problems were also reported in [4, 10]. To improve the efficiency, we suggest in this work to update $L_{u}$ and $L_{\varphi}$ at each iteration $i$ :

$$
L_{i}=a(i) L_{i-1}, \quad \text { where } L_{i}:=L_{u, i}=L_{\varphi, i} .
$$

Inspired by numerical continuation methods in e.g. [1], one would naturally choose a large $L_{0}$ and $a(i):=a<1$ to obtain a decreasing sequence $L_{0}>L_{1}>L_{2}>\ldots$, updated until a lower bound $L_{-}$is reached. However, this seems not to be a good choice in phase-field fracture since the system does not have a unique solution. Consequently, with increasing $i$ the iterations would oscillate in approaching one or another solution, and the algorithm convergence deteriorates. For this reason, we propose the other way around: the closer the iteration is to some solution, the larger the stabilization parameters is chosen, so that the iterations remain close to this solution. We choose $a(i):=a>1$, yielding $L_{0}<L_{1}<L_{2}<\ldots$ up to a maximal $L_{*}$.

On the specific choice of the parameters: A possible choice for $a$ is $a(i):=5^{i}$ $(i=0,1,2, \ldots)$, while $L_{0}:=10^{-10}$. This heuristic choice and may be improved by using the solution within the iteration procedure, or a-posteriori error estimates for the iteration error. Moreover, $a(i):=5^{i}$ is motivated as follows. Higher values greater than 5 would emphasize too much the stablization. On the other hand, too low 
values, do not lead to any significant enhancement of the convergence behaviour. We substantiate these claims by also using $a(i)=10^{i}$ and $a(i)=20^{i}$ in our computations.

Dynamic update using the iteration: An extension of the strategy is to adapt the $L$-scheme parameters in space by using the phase-field variable $\varphi^{n, i-1}$. We still take $L_{i}=a L_{i-1}$, but now $a:=a\left(i, \varphi^{n, i-1}\right)$. Away from the fracture, we have $\varphi \approx 1$ and essentially only the elasticity component (2) is being solved. On the other hand, the stabilization is important in the fracture region, for which we take

$$
L_{i}=a\left(i, \varphi^{n, i-1}\right) L_{i-1}, \quad \text { with } a\left(i, \varphi^{n, i-1}\right):=\left(1-\varphi^{n, i-1}\right) a .
$$

Recalling that the fracture is characterised by $\varphi \approx 0$, it becomes clear that the stabilization parameters are acting mainly in the fracture region. Finally, to improve further the convergence behaviour of the scheme we adapt $\Xi$ at each iteration. In this case we take $\Xi_{i}=\Xi_{i-1}+\gamma\left[\varphi^{n, i-1}-\varphi^{n-1}\right]^{+}$.

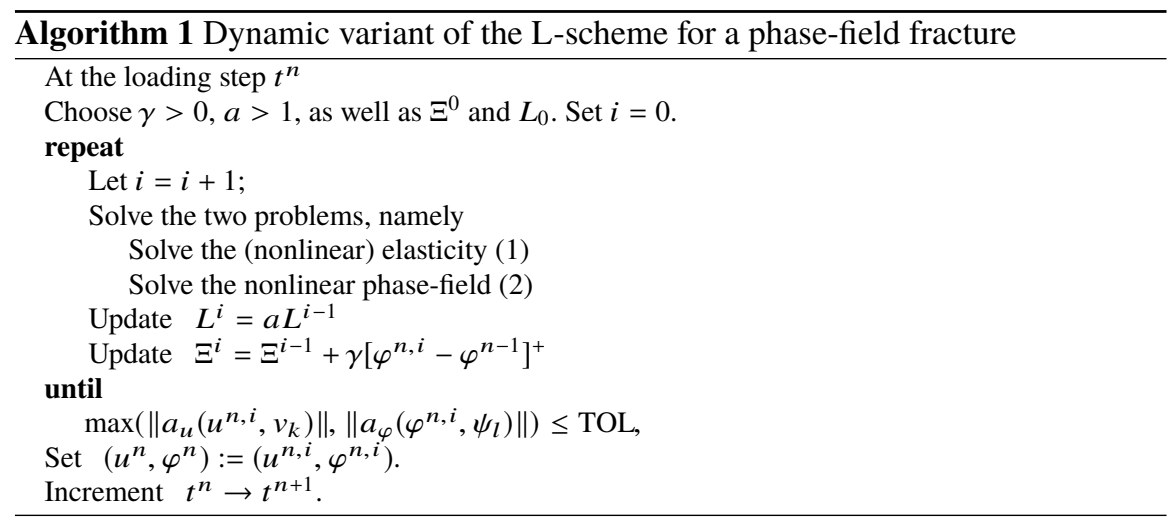

The final algorithm: The algorithm is based on the iterative procedure for phasefield fracture originally proposed in [9]. Therein, the inequality constraint is realized by an augmented Lagrangian iteration. Within this loop we update the $L$ scheme parameters too. The resulting is sketched in Algorithm 3 , in which TOL $=10^{-6}$ is taken, and $L=L_{u}=L_{\varphi}$.

Remark 1. For the solution of both nonlinear subproblems (1) and (2), we use a monotonicity-based Newton method (details see e.g., in [10]) with the tolerance $10^{-8}$. Inside Newton's method, we solve the linear systems with a direct solver.

\section{Numerical tests}

We consider two test examples. Details for the first test van be found in [7]. The setup of the second test can be found for instance in [6]. Both examples were already 
computed in [3] and the results therein are compared to the ones obtained here. The scheme is implemented in a code based on the deal.II library [2].
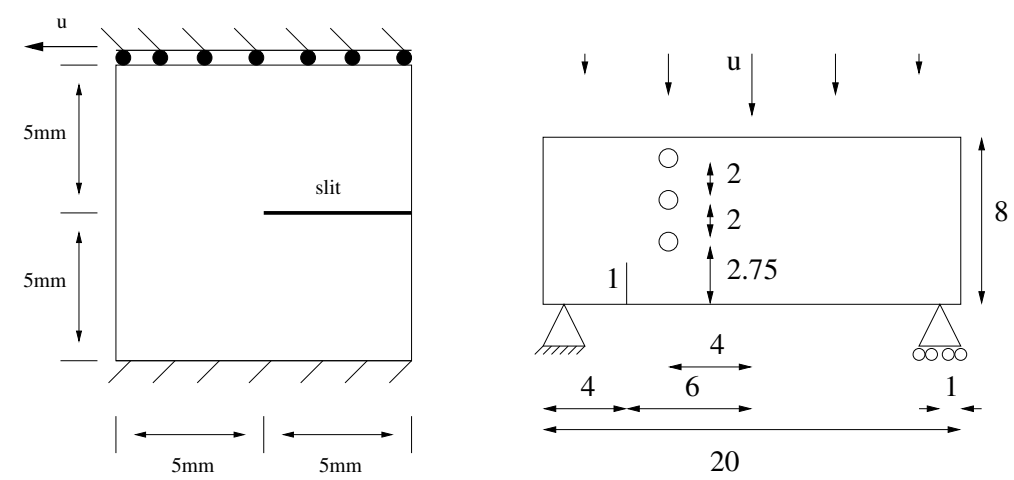

Fig. 1 Examples 1 and 2. The following conditions are prescribed: on the left and right boundaries, $u_{y}=0 \mathrm{~mm}$ and traction-free in $x$-direction. On the bottom part, $u_{x}=u_{y}=0 \mathrm{~mm}$. On $\Gamma_{\text {top }}, u_{y}=$ $0 \mathrm{~mm}$ and $u_{x}$ is as stated in (3). Finally, the lower part of the slit is fixed in $y$-direction, i.e., $u_{y}=$ $0 \mathrm{~mm}$. Right: Asymmetric notched three point bending test. The three holes have each a diameter of 0.5. All units are in $\mathrm{mm}$.
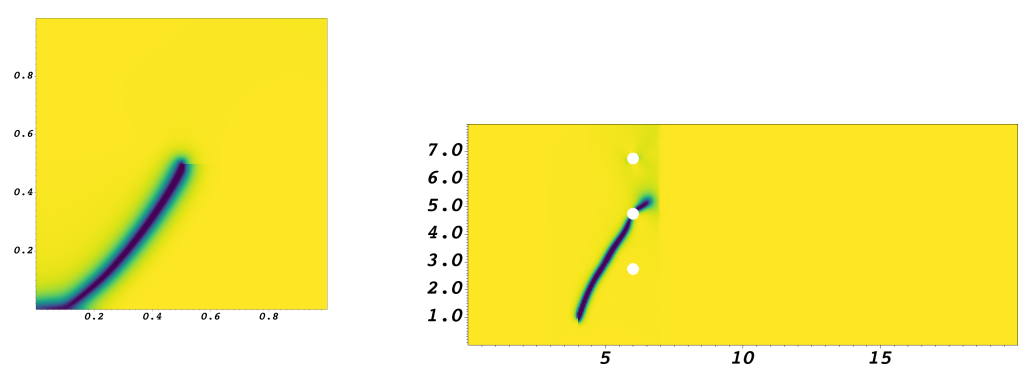

Fig. 2 Examples 1 and 2. Numerical solutions on the finest meshes and at the end time. The cracks are displayed in dark blue color.

Single edge notched shear test: The configuration is shown in Figure 11. Specifically, we use $\mu_{s}=80.77 \mathrm{kN} / \mathrm{mm}^{2}, \lambda_{s}=121.15 \mathrm{kN} / \mathrm{mm}^{2}$, and $G_{c}=2.7 \mathrm{~N} / \mathrm{mm}$. The crack growth is driven by a non-homogeneous Dirichlet condition for the displacement field on $\Gamma_{\text {top }}$, the top boundary of $B$. We increase the displacement on $\Gamma_{\text {top }}$ over time, namely we apply non-homogeneous Dirichlet conditions:

$$
u_{x}=t \bar{u}, \quad \bar{u}=1 \mathrm{~mm} / \mathrm{s}
$$

where $t$ denotes the current loading time. Furthermore, we set $\kappa=10^{-10}[\mathrm{~mm}]$ and $\varepsilon=2 h[\mathrm{~mm}]$. We evaluate the surface load vector on the $\Gamma_{\text {top }}$ as 


$$
\tau=\left(F_{x}, F_{y}\right):=\int_{\Gamma_{\text {top }}} \sigma(u) v \mathrm{~d} s,
$$

with normal vector $v$, and we are particularly interested in the shear force $F_{x}$. Three different meshes with 1024 (Ref. 4), 4096 (Ref. 5) and 16384 (Ref. 6) elements are observed in order to show the robustness of the proposed schemes. The results are shown in Figure6.

Our findings are summarized in Figure 3. The numerical solutions for all four different strategies for choosing $L$ are practically identical, only the number of iterations being different. Here, $L=0$ and $L=1 e-2$ denote tests in which $L=L_{u}=L_{\varphi}$ are taken constant throughout the entire computation. The newly proposed dynamic versions are denoted by L dynamic and L dyn. weighted. We observe a significant reduction in the computational cost when using the dynamic $L$-schemes. The maximum number of iterations is 21 for both the weighted version and the spatially-constant $L$-scheme. This number is reduced to 12 iterations using $a=20$ while the accuracy only slightly changes.
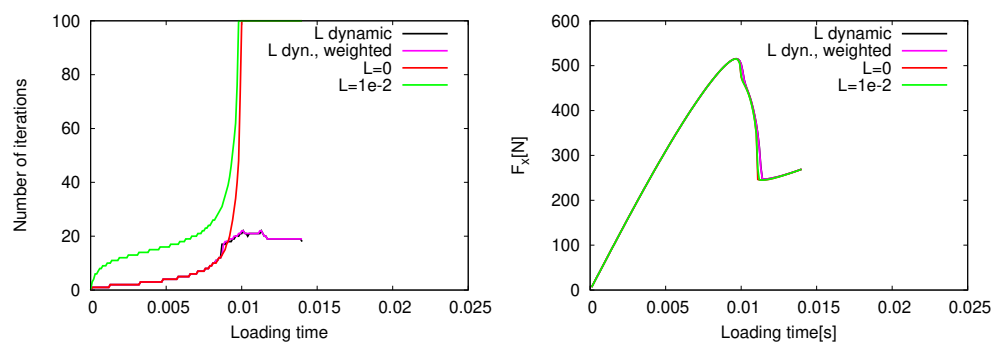

Fig. 3 Example 1. Comparison of dynamic $L$ updates, the weighted version, and constant $L$. Left: number of iterations. Right: load-displacement curves.
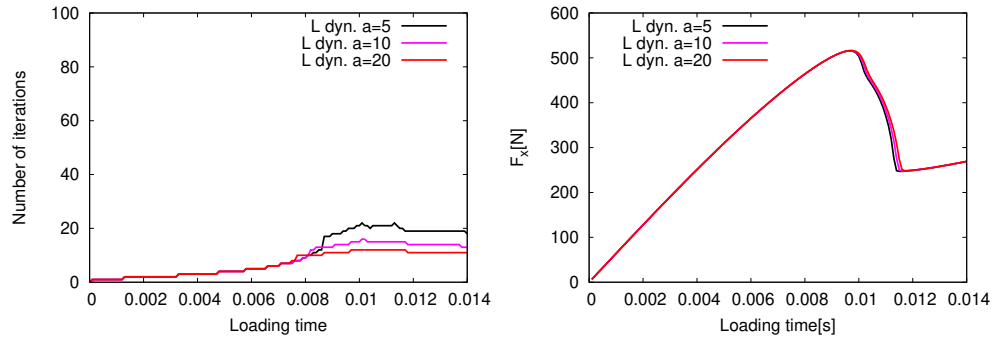

Fig. 4 Example 1. Comparison of different $a$ for the dynamic $L$ scheme. 
Asymmetrically notched three point bending test: The configuration is shown in Figure 1 (right). The initial mesh is 3,4 and 5 times uniformly refined, yielding 3904,15616 and 62464 mesh elements with the minimal mesh size parameter $h_{3}=0.135, h_{4}=0.066$ and $h_{5}=0.033$. As material parameters, we use $\mu_{s}=$ $8 \mathrm{kN} / \mathrm{mm}^{2}, \lambda_{s}=12 \mathrm{kN} / \mathrm{mm}^{2}$, and $G_{c}=1 \times 10^{-3} \mathrm{kN} / \mathrm{mm}$. Furthermore, we set $k=10^{-10} h[\mathrm{~mm}]$ and $\varepsilon=2 h$.

Figure 5 presents the number of iterations and the load-displacement curves. The number of iterations is decreasing from 500 (in the figures cut to 100) for the classical L-scheme, to a maximum of 25 when using the dynamic updates. The choice of weighting does not seem to have a significant influence on the number of iterations though. The crack starts growing a bit later when using the dynamic updates, which can be inferred from the right plot in Figure 5. Thus, the stabilization parameters have a slight influence on the physical solution. This can be explained in the following way. In regions where $\varphi=0$ the solution component $u$ is not uniquely defined. This leads to a sub-optimal convergence behaviour of the L-scheme. With the dynamic L-scheme we regain uniqueness, but at the cost of a slightly modified physical problem.
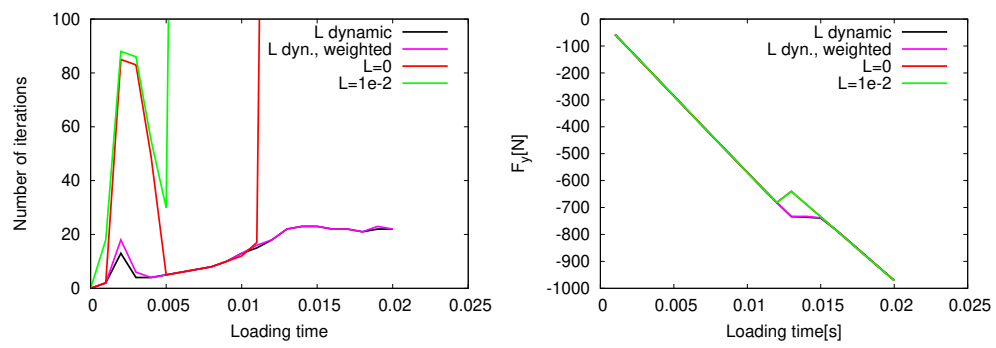

Fig. 5 Example 2: Left: The number of iterations for the different schemes; the results for $L=0$ and $L=1 e-2$ are taken from [3]. Right: The load-displacement curves; a slight difference can be observed in the results, indicating that the dynamic updates lead to a slight delay in the prediction of the starting time for the fracture growth.
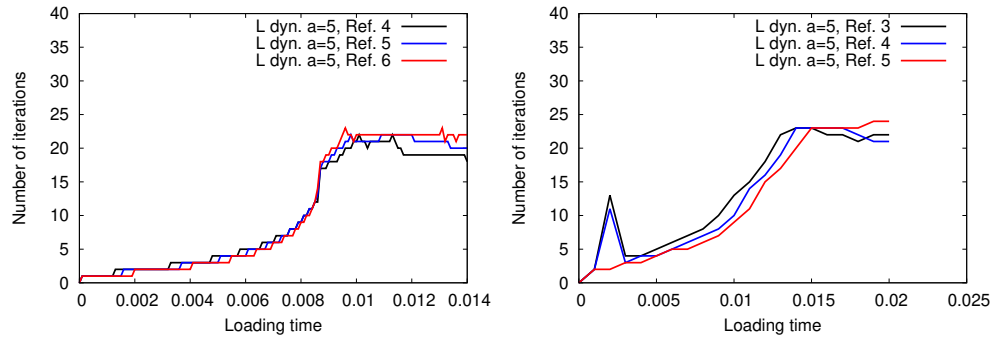

Fig. 6 Examples 1 and 2 for the dynamic $L$ scheme using $a=5$; three different mesh levels are used in order to verify the robustness of the proposed scheme. The results indicate that the mesh size does not influence the number of the iterations. 
Remark 2. Noteworthy, the number of iterations for the dynamic L-scheme is robust with respect to the mesh refinement, as shown in Figure 6 This is in line with the analysis in [3, 5, 8], where it is proved that the convergence rate does not depend on the spatial discretization.

\section{Acknowledgements}

TW is supported by the German Research Foundation, Priority Program 1748 (DFG SPP 1748) under the project No. 392587580. CE is supported by the German Research Foundation, via Priority Program 1648 (DFG SPP 1648) under the grant No. EN-1042/2-2 and via EXC 2044-390685587, Mathematics Münster: Dynamics-Geometry-Structure. ISP is supported by the Research Foundation-Flanders (FWO), Belgium through the Odysseus programme (project G0G1316N).

\section{References}

1. E. L. Allgower and K. Georg. Numerical continuation methods: an introduction. Springer, 1990.

2. D. Arndt, W. Bangerth, T. C. Clevenger, D. Davydov, M. Fehling, D. Garcia-Sanchez, G. Harper, T. Heister, L. Heltai, M. Kronbichler, R. M. Kynch, M. Maier, J.-P. Pelteret, B. Turcksin, and D. Wells. The deal. II library, version 9.1. J. Numer. Math., 2019. accepted.

3. M. K. Brun, T. Wick, I. Berre, J. M. Nordbotten, and F. A. Radu. An iterative staggered scheme for phase field brittle fracture propagation with stabilizing parameters. arXiv preprint arXiv:1903.08717, accepted for publication in Comp. Meth. Appl. Mech. Engrg., 2019.

4. T. Gerasimov and L. D. Lorenzis. A line search assisted monolithic approach for phase-field computing of brittle fracture. Comp. Meth. Appl. Mech. Engrg., 312:276 - 303, 2016.

5. F. List and F. A. Radu. A study on iterative methods for solving Richards' equation. Comput. Geosci., 20(2):341-353, 2016.

6. A. Mesgarnejad, B. Bourdin, and M. Khonsari. Validation simulations for the variational approach to fracture. Comp. Meth. Appl. Mech. Engrg., 290:420 - 437, 2015.

7. C. Miehe, F. Welschinger, and M. Hofacker. Thermodynamically consistent phase-field models of fracture: variational principles and multi-field fe implementations. Int. J. Numer. Methods Engrg., 83:1273-1311, 2010.

8. I. S. Pop, F. Radu, and P. Knabner. Mixed finite elements for the Richards' equation: linearization procedure. J. Comput. Appl. Math., 168(1-2):365-373, 2004.

9. M. Wheeler, T. Wick, and W. Wollner. An augmented-Lagangrian method for the phase-field approach for pressurized fractures. Comp. Meth. Appl. Mech. Engrg., 271:69-85, 2014.

10. T. Wick. An error-oriented Newton/inexact augmented Lagrangian approach for fully monolithic phase-field fracture propagation. SIAM J. Sci. Comput., 39(4):B589-B617, 2017. 